\title{
An Exploration Of Geographic Scope: The Cluster Of Grenoble
}

Laurent Scaringella, ESC Rennes School of Business, France

\begin{abstract}
This article examines the high-tech cluster of Grenoble in the light of regional studies. In particular, we explore the geographic scope of organizations, knowledge flows and risk perceptions. Using a large quantitative dataset, we observe that trial-driven synthetic knowledgeflow dynamics are generally based on the engineering sciences and develop over large distances, posing a challenge to well-established clusters. Our results emphasize significant differences across organization types (firms, research centers, universities, and public bodies) and organization sizes (small, medium, and large). We find that large firms develop knowledge-flows dynamics over greater distances than small firms and that research centers, universities and medium-sized firms perceive greater knowledge anchoring than do small and large firms. In addition, we find that theory-driven analytical and branding-driven symbolic knowledge are more anchored than synthetic knowledge, which is the type of knowledge of greatest value in information and communication technologies (ICT). Finally, we argue that the increase of geographical distance between knowledge senders and receivers increases the perception of the risk of unintended knowledge spillovers.
\end{abstract}

Keywords: Knowledge; Proximity; Distance; Cluster

\section{INTRODUCTION}

eographical distance between knowledge senders and receivers is no longer a technical barrier to knowledge transfer. However, the increasing mobility of knowledge raises questions about the geographic scope of organizations and their ability to manage this increased knowledge mobility. It also raises questions regarding the degree of compatibility between the particular type of knowledge an organization uses and/or creates and small or large geographical distances. Finally, organizations may legitimately worry that longdistance knowledge-flow dynamics carry greater risk of unintended knowledge spillovers than knowledge-flow dynamics developed locally. All these questions both fuel and challenge existing research on clusters grounded in agglomeration theory. The present article therefore asks, "What is the geographic scope of organizations, knowledge and unintended spillovers within the cluster of Grenoble?"

We employ an exploratory quantitative approach in examining the cluster of Grenoble. Focusing particularly on geographical proximity/distance, knowledge anchoring/mobility and the risk of unintended spillovers, a questionnaire was completed by 932 highly educated individuals from 111 firms, research centers, universities, and public bodies in the cluster of Grenoble. We used descriptive statistics to obtain a "first look" at the results.

We begin by presenting the theoretical framework of the study, highlighting the need for further exploration of regional clusters. The methods employed in the study are then presented, followed by a presentation of the results and a discussion. A final section concludes, and suggestions for further study are presented.

\section{THEORETICAL FRAMEWORK}

In the field of regional studies, there has been a paradigm shift in focus from national to regional innovation mechanisms. Two decades ago, Porter (1990a) argued that countries have important leverage over their innovation 
capabilities. In particular, the notion of the National System of Innovation (NSI) was developed by Freeman (1987), while the notion of the National Innovation System (NIS) was developed by Nelson (1993) and Lundvall (1992). The central mechanism identified in these theories was national institutions as a driving force of innovation (Nelson, 2000) that allowed for a "network of institutions in the public and private sectors whose activities and interactions initiate, import, modify and diffuse new technologies" (Freeman, 1987).

More recently, innovation at the regional level has followed the pattern of well-established national systems. In both national and regional settings, a variety of organizations are involved: firms, research centers, universities and public institutions - organizations that are increasingly collaborating and sharing knowledge within and across regions, causing a shift, according to Cooke (2003) and Malmberg \& Maskell (2002), toward Regional Innovation Systems (RIS). This growing importance of the regional economy has encouraged researchers to develop several conceptual frameworks called "Territorial Innovation Models" (TIMs) to better understand the reasons behind the disparities between flourishing regions and regions that have slowed down. TIMs combine models, such as innovative milieu, industrial districts, localized production systems, new industrial spaces, innovation clusters, regional innovation systems and learning regions (Moulaert \& Sekia, 2003).

TIMs depict geographical concentrations and connections among companies, suppliers, service providers, firms, and institutions (Porter, 1998). They are based on agglomeration theory, first developed by Alfred Marshall in the $19^{\text {th }}$ century. Marshall (1920) studied the economies of scale of firms established in industrial districts that offer proximity networking to other firms, suppliers, and clients, enabling firms to benefit from knowledge spillovers that reduce costs. To illustrate the effects of agglomeration, Marshall used as an example the cutlery industry in the Sheffield area where "the mysteries of the trade become no mysteries, but are as it were in the air." Grounded in the notion of the division of labor, regions become specialized and interact economically with others regions. Collaborations thus form to develop composite products based on regional specializations.

The agglomeration effect at the cluster level is based on the intensity of knowledge-flow dynamics both within and outside the cluster. Unfortunately, the literature does not distinguish between organizations in terms of geographic scope. Because the scope of interaction is organization-specific, this represents a theoretical weakness, as, for example, a typical firm will have a different geographic scope than a typical research center. Similarly, existing TIMs do not explore differences in geographic scope between large international firms and start-ups. Thus, the present article explores the geographic scope of the cluster of Grenoble and most of its members. At the cluster level, we expect that geographical distance will generally be important because competition in the information and communication technologies (ICT) sector is global. However, we expect some differences between organizations. For example, research centers, universities and public bodies should be more involved in local networks, while firms should be more involved in global networks. We also expect to observe differences between organizations with respect to knowledge anchoring and mobility, depending on the type and size of an organization.

Following this initial enquiry, the article will explore geographic scope as it pertains to the three categories of knowledge defined by Cooke (2005): analytical, synthetic, and symbolic knowledge. Analytical knowledge is science-based, i.e., theory-driven (Laestadius, 2000), and requires the involvement of multiple stakeholders in both industry and research. Such cooperation leads to a high level of proximity within science parks, technical universities, and so forth (Asheim \& Coenen, 2004). Analytical knowledge is developed within communities of practice located in limited geographical spaces, which increases feelings of trust among members. According to Cooke (2005), such proximity leads to the development of centers of excellence based around leading universities and firms. Consequently, we expect to observe mobilization of analytical knowledge primarily within knowledgeflow networks over relatively close geographical distances. Different in nature from analytical knowledge, synthetic knowledge is based on engineering within the industrial sector. According to Laestadius (2000), synthetic knowledge is trial-driven and codified to facilitate knowledge transfer at a distance. Thus, we expect to observe stronger mobilization of synthetic knowledge within knowledge-flow dynamics occurring over greater distances than we observe in the case of analytical knowledge. Finally, symbolic knowledge is characterized by a high degree of art, communication, and branding (Cooke, 2005). Firms seek to develop such knowledge to improve their image and create unique value for their customers. In practice, regional affiliation strongly affects product image. From a consumer perspective, the quality of a product is typically associated with its region of design and/or manufacture. For example, consumers commonly believe that good cars are made in Germany and that good watches are made in 
Switzerland. In the present article, we expect to observe stronger mobilization of symbolic knowledge within knowledge-flow dynamics occurring in close geographical proximity.

The third issue examined in this article concerns the link between the geographic scope of knowledge-flow dynamics and the risk of unintended knowledge spillovers. Based on the current expansion of long-distance knowledge flows, some scholars and practitioners have noted a growing risk of unintended knowledge spillovers. Focusing particularly on the ICT sector, Brossard \& Vicente (2007) argued that such risk is unrelated to geographical proximity and distance. Challenging the concept of geographical proximity, the authors based their analysis on the correlation between cognitive proximity and spillover risk. According to their argument, long distance knowledge flow is no more risky than local knowledge flow. Consequently, we expect to find no correlation between the risk of unintended knowledge spillover and geographical distances between organizations.

\section{DATA AND METHODS}

Following the section on the theoretical framework used in the study, we employ a quantitative approach to explore the three axes referenced above: the geographic scope of the cluster and its organizations, the geographic scope of knowledge types, and the geographic scope of unintended knowledge spillovers. To initiate the discussion, the article focuses on the particular technology cluster of Grenoble.

Located in the French Alps $550 \mathrm{~km}$ from Paris and $100 \mathrm{~km}$ from Lyon, Grenoble benefits from a somewhat secluded environment amidst mountains while retaining connections with the rest of the world. Considered a hightech cluster, Grenoble specializes in Information Technologies (IT), micro/nanotechnologies, renewable energy, and biotechnology. Over the last 15 years, Grenoble has seen a significant expansion of the IT sector as a result of cumulations of knowledge in physics, chemistry and materials and is now turning to renewable energy and biotechnology as a result of composite knowledge flows across fields. Grenoble has 435,400 inhabitants, a high proportion of whom are highly educated, and is located in one of the most dynamic and profitable regions in Europe. Grenoble benefits from the concentration of complementary organizations and substantial network thickness.

To conduct the study, we administered an online survey. Of 5,000 people contacted, 932 responded (a response rate of $18.64 \%$ ). Of those who responded, $77.2 \%$ completed the survey in full. The quantitative data yielded by the survey enabled us to compute descriptive statistics. The design of the questionnaire was based on a literature review and two perception items: the perception of geographical proximity/distance between knowledge senders and receivers and the perception of knowledge anchoring/mobility.

At the cluster level, various types of organizations involved in the knowledge value chain were surveyed: firms $(51 \%)$, research centers $(25 \%)$, universities $(18 \%)$, and public bodies $(6 \%)$. Among firms, the size of an organization matters and may influence the geographic scope of knowledge-flow dynamics. Therefore, we created three categories: small-sized organizations (1-10 people), medium-sized organizations (10-500 people), and largesized organizations (more than 500 people). In total, 111 organizations contributed to the survey. At the individual level, data on the functional roles of respondents, their education levels and management power (number of subordinates) were collected. The sample is not representative of the region because we only targeted knowledgeintensive individuals. Consequently, 836 survey respondents (91.5\% of the sample) hold managerial and/or highly intellectual jobs, while employees and workers represent only $7.7 \%$ of the sample. Job profile is strongly correlated with education level in the sample. Indeed, half the respondents hold master's degrees, and $37 \%$ hold PhDs, while just $13 \%$ hold only undergraduate degrees. A simple computation reveals that the number of years of study past high school over the sample reaches an impressive 5,350 years of study, with an average of 5.85 years of education per individual following high school. Such an average is not representative of national statistics but emphasizes the gap between national figures and those of technology clusters such as Grenoble. Management power not related to educational level remains low. Of the respondents, 32\% do not have any employees under their responsibility, and $30 \%$ manage between one and five individuals. This indicates that people working in research centers or universities are highly educated but do not necessarily have subordinates. With respect to the gender balance, $69.1 \%$ of the respondents were male, and $30.9 \%$ were female, which matches the proportion of males and females in this sector. 


\section{RESULTS AND DISCUSSION}

Based on the data collected, we seek to gain a better understanding of the geographic scope of knowledgeflow dynamics within and between organizations. Our objectives are, first, to identify the general geographic scope (distant versus proximate knowledge-flow dynamics) of the cluster and, second, to determine whether there are differences among organizations. Generally, knowledge-flow dynamics are established over distances. Such distance arises from the nature of the ICT sector, which is highly competitive and globally established. As knowledge has become a scarce resource, the search for rich knowledge that may be distantly located has intensified. International firms (IFs) differ significantly from other stakeholders in that they establish knowledge-flow dynamics over large distances to obtain knowledge from different locations. In contrast, medium-sized firms (MFs), small-sized firms (SFs), research centers (RCs), and universities (UNs) do not differ significantly in terms of the geographic distances spanned by their knowledge-flow networks. Finally, the geographic distance/proximity of such organization differs significantly from that of public bodies (PBs), which have local connections to the cluster of Grenoble. Such proximity reinforces local anchoring through the creation of a unique innovation-enhancing environment.

Based on the geographic scope of knowledge exchange, the present article seeks to measure perceptions of knowledge anchoring/mobility. The empirical results present a picture of knowledge that is generally more mobile than anchored. However, there are significant differences across organizations. IFs and SFs exhibit similar patterns, i.e., mobile knowledge. Statistically, medium-sized companies differ significantly from international and small firms, with MFs perceiving stronger knowledge anchoring within the local economy. Their technological development is often targeted toward market niches, allowing them to create differentiated products and services that are relatively protected from global competition. Their areas of specialization are based on core knowledge concentrated within the cluster of Grenoble. Similarly, RCs differ significantly from IFs, perceiving knowledge to be strongly anchored within the local economy. Knowledge developed by UNs offers a dual perspective: halfanchored (through links with RCs) and half-mobile (through dissemination of knowledge via publications and conferences). Finally, PBs develop anchored knowledge to meet local organizations' needs.

Based on the domination of long-distance knowledge-flow dynamics and knowledge mobility, the article will determine whether the nature of knowledge (analytical, synthetic, and symbolic) is linked to geographic scope. In the cluster of Grenoble, synthetic knowledge is the most important. However, analytical knowledge is also strong, while symbolic knowledge is underdeveloped, as the ICT sector focuses more on technical performance than on image.

In examining the relationship between the mobilization of different types of knowledge and the geographic scope of knowledge flows, we find that knowledge-flow dynamics tend to encompass large distances for all types of knowledge. Contrary to expectations based on the literature, statistically, there is no significant difference between analytical, synthetic and symbolic knowledge in this respect. For example, analytical knowledge flows do not tend to be geographically proximate, and synthetic knowledge flows do not tend to be geographically distant. However, the stronger the mobilization is of analytical knowledge, the stronger is anchoring (although the average score for anchoring does not exceed 4 out of 7). Synthetic knowledge is slightly less anchored than analytical knowledge. Such mobility can be partially explained by the global scope of engineering knowledge flows. Anchoring is stronger for symbolic knowledge than for the other types of knowledge. This implies that the construction of a brand image for the cluster would reinforce its anchoring and economic robustness, a finding that may encourage the cluster to avoid neglecting this type of knowledge.

Given increasing geographical distances of knowledge mobility, the article also points to limits of such a trajectory. The risk of unintended knowledge spillovers is a major concern for organizations. Our empirical results suggest that concern about the level of unintended knowledge spillover increases, from "high" to "very high," with distance between knowledge senders and receivers. Locally, such risk appears to be slightly less, perhaps as a result of greater trust, more knowledge intensive interactions and the development of relational proximity through informal discussions within communities of practice. However, based on our measurements, it is evident that the sense of risk is generally high for any geographic distance. This perception of "high" or even "very high" risk does not discourage organizations from intensifying knowledge-flow dynamics globally in developing strategic alliances, expanding their activities into other industries and competing globally. 


\section{CONCLUSION}

Based on a review of the regional studies literature, we have identified a need to examine the geographic scope of knowledge-flow dynamics from three perspectives: organization, knowledge, and risk. In the cluster of Grenoble, which specializes in high-tech industries, synthetic knowledge-flow dynamics have expanded over a large geographical distance, challenging knowledge anchoring within the cluster. This geographical expansion has significantly affected perceptions of the risk of unintended knowledge spillovers.

The large quantity of data collected yield the following findings. For organizations, the geographic scope of knowledge-flow dynamics emphasizes long distances between large firms and partners. Slightly less distant small- and medium-sized firms, research centers, and universities share similar perceptions of geographic scope, whereas public bodies are more locally focused. Knowledge mobility is strong among large and small companies. Medium-sized companies, by contrast, view knowledge - for example, knowledge based in research centers and universities - as less mobile. Among different types of knowledge, synthetic knowledge is dominant in exchanges, followed by analytical and symbolic knowledge. Our results show that both analytical and symbolic knowledge are more anchored than synthetic knowledge, which is more mobile. From a risk perspective, the greater the geographic distance that knowledge must traverse, the greater the perception of risk of unintended knowledge spillovers.

The present study encounters some limits related to the use of subjective rather than objective measures. We also recognize that the cluster of Grenoble is sector- and region-specific and thus not fully representative. To overcome the issue of subjectivity, we recommend that scholars undertake complementary studies using Social Network Analysis. In further studies, we also suggest examining the relationship between the geographic scope of organizations and their size. In the high-tech sector, we conjecture the existence of an optimum level of knowledge anchoring for medium-sized firms. In the same vein, it would be helpful to understand what other parameters (predictor and effect) affect the development of long- versus short-distance knowledge-flow dynamics.

\section{ACKNOWLEDGEMENTS}

I would like to thank Professor Jean-Jacques Chanaron, the Project Director of the Grenoble EURODITE research team, Professor Emeritus Bernard Chapelet, the former director of the TIME center and the numerous people active in the European research program EURODITE, for their advice and guidance.

\section{AUTHOR INFORMATION}

Laurent Scaringella is an assistant professor at the ESC Rennes School of Business and a visiting scholar at the Haas School of Business at the University of California, Berkeley. E-mail: laurent.scaringella@esc-rennes.fr

\section{REFERENCES}

1. Asheim, B. T., \& Coenen, L. (2004). The role of Regional Innovation Systems in a globalising economy: Comparing Nordic clusters. Philadelphia.

2. Brossard, O., \& Vicente, J. (2007). Knowledge trends in the European ICT Sector, knowledge value chain, proximities and networks. Eurodite.

3. Cooke, P. (2003). The evolution of biotechnology in three continents: Schumpeterian or Penrosian. European Planning Studies, 11(7), 789-804.

4. Cooke, P. (2005). Markets and networks in the knowledge value chain. EU-FP6 EURODITE.

5. Freeman, C. (1987). Technology policy and economic performance: Lessons from Japan. London: Pinter.

6. Laestadius, S. (2000). Biotechnology and the potential for a radical shift of technology in forest industry, 12.

7. Lundvall, B. A. (1992). National systems of innovation: Towards a theory of innovation and interactive learning. London: Pinter.

8. Malmberg, A., \& Maskell, P. (2002). The elusive concept of localization economies: Towards a knowledge-based theory of spatial clustering. Environment and Planning A, 34(3), 429-449. 
9. Marshall, A. (1920). Principles of economics (8th ed.). London, Philadelphia: Macmillan, Porcupine Press.

10. Nelson, R. R. (1993). National systems of innovation: A comparative study. Oxford: Oxford University Press.

11. Nelson, R. R. (2000). National innovation systems. In Z. Acs (Ed.), Regional innovation, knowledge and global change (pp. 11-26). London: Pinter.

12. Porter, M. E. (1990). The competitive advantage of nations. Harvard Business Review, 68(2), 73-93.

13. Porter, M. E. (1998). Cluster and the new economics of competition. Harvard Business Review, 76(6). 